University of Wollongong

Research Online

Faculty of Engineering - Papers (Archive)

Faculty of Engineering and Information

Sciences

November 1995

\title{
Magnetoplasmon emission versus Landau-level scattering in resonant tunneling through double-barrier structures
}

C. Zhang

University of Wollongong, czhang@uow.edu.au

D. J. Fisher

University of Wollongong

Follow this and additional works at: https://ro.uow.edu.au/engpapers

Part of the Engineering Commons

https://ro.uow.edu.au/engpapers/260

\section{Recommended Citation}

Zhang, C. and Fisher, D. J.: Magnetoplasmon emission versus Landau-level scattering in resonant tunneling through double-barrier structures 1995.

https://ro.uow.edu.au/engpapers/260

Research Online is the open access institutional repository for the University of Wollongong. For further information contact the UOW Library: research-pubs@uow.edu.au 


\title{
Magnetoplasmon emission versus Landau-level scattering in resonant tunneling through double-barrier structures
}

\author{
C. Zhang and D. J. Fisher \\ Department of Physics, University of Wollongong, New South Wales 2522, Australia
}

(Received 29 June 1995)

\begin{abstract}
We consider electron tunneling in a parallel magnetic field taking into account the electron-electron interaction. It is shown that a self-consistent treatment of the dynamical electron-electron interaction in resonant tunneling can lead to the proposed plasmon assisted resonant tunneling. Such magnetoplasmon assisted resonant tunneling gives rise to satellite peaks or shoulders in the tunneling current. At low temperatures, only magnetoplasmon emission processes contribute and satellites only appear on the high bias side of the main resonance. The mechanism proposed here may be used to study the magnetotunneling in high mobility systems where disorder is at minimum.
\end{abstract}

\section{INTRODUCTION}

Double-barrier resonant tunneling structures (DBRTS's) represent interesting physical systems with which several fundamental properties related to quantum transport in small structures can be studied. Some well known phenomena in DBRTS's are negative differential resistance, ${ }^{1,2}$ intrinsic bistability,$^{3-5}$ and tristabilities. ${ }^{6,7}$ These phenomena, together with the fast response time in DBRTS's, make these structures a promising class of materials for the next generation electronic devices. Recently, an electron tunneling mechanism in DBRTS's was observed and reported. ${ }^{8}$ When an asymmetric DBRTS is biased in such a way that large charge accumulation occurs in the resonant well, an additional tunneling channel can become available, which is classified as plasmon assisted resonant tunneling (PAT). PAT occurs when, near the resonant tunneling, an incoming electron lowers its energy to the resonant level by emitting a quantum-well plasmon. Due to the nature of quantum-well plasmon dispersion, such processes can occur near the main resonance, as long as the difference between the incoming energy and the resonant level is smaller than the maximum plasmon energy. As a result, a satellite is observed in the tunneling $I-V$ characteristics. If one further includes the static level shift and treats both level shift and PAT self-consistently ( via $n_{2 \mathrm{D}} \sim I$, where $I$ is the tunneling current), the resulting $I-V$ curve exhibits multiple stabilities.

When a magnetic field parallel to the tunneling current is applied to the system, more interesting tunneling phenomena can occur. ${ }^{9}$ On the high bias side of the main resonance peak, a series of side resonances will appear. The spacing between the successive resonance is roughly the same for fixed magnetic field and increases approximately linearly with the magnetic field strength. This phenomenon is commonly understood within the picture of inter-Landau-level elastic scattering. This elastic scattering process can be described as

$$
\varepsilon_{z}+\varepsilon_{n}=\varepsilon_{z}^{\prime}+\varepsilon_{n^{\prime}}
$$

where $\varepsilon_{z}$ and $\varepsilon_{n}$ are, respectively, the energy in the tunneling direction and the Landau-level energy in the plane perpendicular to the tunneling direction. Therefore, one may observe many side resonances at energy $\varepsilon_{z}+\left(n-n^{\prime}\right) \hbar \omega_{c}$, where $\omega_{c}$ is the cyclotron frequency. This elastic scattering mechanism can successfully explain the observed side resonances. Here, we would like to comment on two points concerning this elastic scattering mechanism. (1) For this mechanism to work, one needs to assume the existence inside the resonant well of a large scatterer concentration. Such an assumption is usually justified for realistic systems considering unavoidable disorders and interface roughness in these structures. However, one should pay special attention when this mechanism is applied to high mobility samples, where disorder is at minimum. (2) It is not understood why there is no side resonance at all on the low bias side of the main peak especially considering that the scattering cross sections for $(n \rightarrow n \pm 1)$ are roughly the same. [It is understood that the process $(n \rightarrow n-1)$ is forbidden if only the lowest Landau level in the emitter is occupied.]

In this paper, we shall propose a different type of resonant tunneling in the presence of a parallel magnetic field $(\mathbf{B}|| \mathbf{I})$. We consider a tunneling process that involves emission and absorption of magnetoplasmons. When the tunneling electron reaches the center well, it is hot and in nonequilibrium. Due to the strong coupling of the tunneling electron with the Fermi sea in the well (if it is charged) or in the emitter (always charged), it can excite a magnetoplasmon and change its energy to match the resonant condition. A schematic diagram of this process is shown in Fig. 1. The mechanism proposed here does not assume any additional scatterers in the well or at the interface. At low temperature, the plasmon population is negligible and only plasmon emission is possible. As a result, one only observes side resonances on the high bias side of the main resonance. If coherence breaking processes are negligible, the spacing between the side resonances will be approximately the same and will depend approximately linearly on the magnetic field. However, it should be pointed out that due to the nature of magne- 


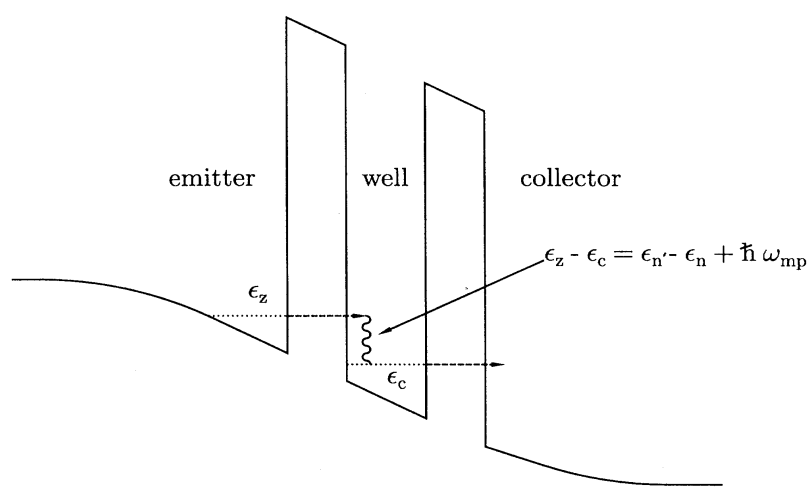

FIG. 1. Schematic diagram for magnetoplasmon assisted resonant tunneling in a double-barrier resonant tunneling structure.

toplasmon dispersion, the spacing is always nonlinear. In this work, we shall assume that the charge accumulation in the well is negligible (such as the case of a symmetric DBRTS or an asymmetric DBRTS of thicker emitter barrier) and shall only consider plasmon excitation in the emitter. The tunneling rate presented below is also valid for the case where plasmon excitation occurs in the center well. However, in case of a charged well, one has to include the dynamical resonance level shift, which causes the tipover of the resonant curve. ${ }^{8,10}$

\section{CALCULATION OF THE TUNNELING RATE}

Let us consider a typical DBRTS (symmetric or asymmetric). The model Hamiltonian can be written as

$$
H=H_{0}+H_{I},
$$

where $H_{0}$ is the one-body Hamiltonian describing the single particle energy, including elastic coupling with the static barriers,

$$
\begin{aligned}
H_{0}= & \sum_{n, k_{y}} \epsilon_{n}^{c} c_{n, k_{y}}^{\dagger} c_{n, k_{y}}+\sum_{\mathbf{p}, \nu} \epsilon_{\mathbf{p}}^{\nu} a_{\mathbf{p} \nu}^{\dagger} a_{\mathbf{p}, \nu} \\
& +\sum_{\mathbf{p}, \nu} V_{\mathbf{p}, \nu}\left(a_{\mathbf{p}, \nu}^{\dagger} c_{n, k_{y}}+c_{n, k_{y}}^{\dagger} a_{\mathbf{p}, \nu}\right)
\end{aligned}
$$

In the above equation, $\mathbf{p}=\left(n, k_{y}, k_{z}\right), \epsilon_{n}^{c}=\epsilon_{c}+(n+$ $1 / 2) \hbar \omega_{c}$, with $\epsilon_{c}$ the energy of quasibound states in the well and $n$ the Landau level (LL) index. $c_{n, k_{y}}^{\dagger}\left(c_{n, k_{y}}\right)$ is the creation (annihilation) operator for electrons in the resonant well with the LL index $n$ and center coordinate $x_{0}=k_{y} / e B$. Similarly, $\epsilon_{\mathbf{p}}^{\nu}=\epsilon_{z}^{\nu}+\epsilon_{n}^{\nu}$, with $\nu=l(r)$ for the left lead (right lead). $a_{\mathbf{p}}^{\dagger}\left(a_{\mathbf{p}}\right)$ is the creation (annihilation) operator for electrons in the leads. $V_{\mathbf{p}}^{\nu}$ is the elastic coupling between the electrons and the static barrier.

When considering the electron-electron interaction, we shall consider that (i) the only important interaction is the interaction between the tunneling electron (after it leaves the emitter) with those electrons in the emitter and the charge concentration in the emitter is taken to be constant. (ii) The interaction among electrons in the emitter is constant if the electron concentration in the emitter is considered to be constant. Such a constant energy shift is unimportant in the present model. (iii) There is no charge accumulation in the well. Now the interaction Hamiltonian is given as

$$
H_{I}=\sum_{n n^{\prime}, k_{y} k_{y}^{\prime}, q} v_{q} C_{n n^{\prime}}(q) c_{n, k_{y}+q_{y}}^{\dagger} a_{n^{\prime}, k_{y}^{\prime}-q_{y}}^{\dagger} a_{n, k_{y}^{\prime}} c_{n, k_{y}},
$$

where $v_{\boldsymbol{q}}=$ is the Fourier coefficient of Coulomb interaction between the tunneling electron in the well and electrons in the emitter. The form factor is given as (for $\left.n>n^{\prime}\right), C_{n n^{\prime}}(q)=\left(n^{\prime} ! / n !\right) X^{n-n^{\prime}} e^{-X}\left[L_{n^{\prime}}^{n-n^{\prime}}(X)\right]^{2}$, where $X=q^{2} / 2 e B$ and $L_{n}^{m}$ is an associated Laguerre polynomial.

The calculation of transmission through a DBRTS is carried out within the so-called $S$-matrix formalism. ${ }^{11-14}$ Let $T\left(\mathbf{p}, \mathbf{p}^{\prime}\right)$ be the transmission matrix describing the probability per unit final longitudinal momentum $k_{z}^{\prime}$, that a particle incident with energy $\epsilon_{\mathbf{p}}$ will be transmitted with energy $\epsilon_{\mathbf{p}^{\prime}}$. The total transmission probability $T_{t}(\mathbf{p})$ with initial momentum $\mathbf{p}$ can be written as a sum (or integral) of the transmission matrix $T\left(\mathbf{p}, \mathbf{p}^{\prime}\right)$ over the final momentum $\mathbf{p}^{\prime}$,

$$
T_{t}(\mathbf{p})=\int d \mathbf{p}^{\prime} T\left(\mathbf{p}, \mathbf{p}^{\prime}\right) .
$$

The transmission matrix can be written as

$$
\begin{aligned}
T\left(\mathbf{p}, \mathbf{p}^{\prime}\right)= & \Gamma_{l}(\mathbf{p}) \Gamma_{r}\left(\mathbf{p}^{\prime}\right)\left|\frac{\partial \epsilon_{\mathbf{p}^{\prime}}}{\partial k_{z}^{\prime}}\right| \int \frac{d \tau d s d t}{2 \pi \hbar^{3}} \exp \left\{i \left[\left(\epsilon_{\mathbf{p}}\right.\right.\right. \\
& \left.\left.\left.-\epsilon_{\mathbf{p}^{\prime}}\right) \tau+\epsilon_{\mathbf{p}^{\prime}} t-\epsilon_{\mathbf{p}} s\right] / \hbar\right\} G_{\alpha, \alpha^{\prime}}(\tau, s, t)
\end{aligned}
$$

Here, $\mathbf{p}\left(\mathbf{p}^{\prime}\right)$ is for the left (right) lead, $\alpha=\left(n, k_{y}\right)$ and $\int d \mathbf{p} \rightarrow \sum_{n} \int d k_{z} \int d k_{y}$. The elastic resonant width is

$$
\Gamma_{l}(\mathbf{p})=2 \pi \sum_{\mathbf{p}^{\prime \prime}}\left|V_{\mathbf{p}^{\prime \prime}, l}\right|^{2} \delta\left(\alpha, \alpha^{\prime \prime}\right) \delta\left(\epsilon_{\mathbf{p}}-\epsilon_{\mathbf{p}^{\prime \prime}}^{l}\right)
$$

with a similar expression for $\Gamma_{\boldsymbol{r}}\left(\mathbf{p}^{\prime}\right)$. The interacting twobody Green's function is defined as

$$
G_{\alpha, \alpha^{\prime}}(\tau, s, t)=\theta(s) \theta(t)\left\langle c_{\alpha}(\tau-s) c_{\alpha^{\prime}}^{\dagger}(\tau) c_{\alpha^{\prime}}(t) c_{\alpha}^{\dagger}(0)\right\rangle
$$

The leading terms in a perturbation expansion of $G_{\alpha, \alpha^{\prime}}$ are given by those up to the second order of the electronelectron interaction. The one-body Green's function is simply $G_{\alpha}^{0}(t)=-i \theta(t) \exp \left[-\left(i \epsilon_{n}+\Gamma / 2\right) t / \hbar\right]$. In the interaction picture, the two-body Green's function is given as 


$$
\begin{aligned}
G_{\alpha, \alpha^{\prime}}(\tau, s, t)= & \theta(s) \theta(t)\left\langle\hat{c}_{\alpha}(\tau-s) U^{\dagger}(\tau\right. \\
& \left.-s) \hat{c}_{\alpha^{\prime}}^{\dagger}(\tau) \hat{c}_{\alpha^{\prime}}(t) U(t, 0) \hat{c}_{\alpha}^{\dagger}(0)\right\rangle,
\end{aligned}
$$

with $U\left(t, t_{0}\right)$ the time evolution operator,

$$
U\left(t, t_{0}\right)=\sum_{m=0}^{\infty} T\left[-\imath \int_{t_{0}}^{t} d t^{\prime} \hat{H}_{\mathrm{int}}\left(t^{\prime}\right)\right]^{m} / m !
$$

To the second order, it can be written as

$$
\begin{aligned}
G_{n, k_{y} ; n, k_{y}^{\prime}}^{(2)}(\tau, s, t)= & \theta(s) \theta(t)\left\langle\hat{c}_{n, k_{y}}(\tau-s)\left(\imath \int_{\tau-s}^{\tau} d t^{\prime} \hat{H}_{\mathrm{int}}\left(t^{\prime}\right)\right) \hat{c}_{n^{\prime}, k_{y}^{\prime}}^{\dagger}(\tau) \hat{c}_{n^{\prime}, k_{y}^{\prime}}(t)\left(-\imath \int_{0}^{t} d t^{\prime} \hat{H}_{\mathrm{int}}\left(t^{\prime}\right)\right) \hat{c}_{n, k_{y}}^{\dagger}(0)\right\rangle \\
= & \theta(s) \theta(t) \sum_{n_{i}, n_{i}^{\prime}, k_{y i}, k_{y i}^{\prime}, q_{i}} \int_{\tau-s}^{\tau} d s^{\prime} \int_{0}^{t} d t^{\prime} v_{q_{1}} v_{q_{2}} C_{n_{1} n_{1}^{\prime}}\left(q_{1}\right) C_{n_{2} n_{2}^{\prime}}\left(q_{2}\right) \\
& \times\left\langle\hat{c}_{n, k_{y}}(\tau-s) \hat{c}_{n_{1}, k_{y 1}+q_{y 1}}^{\dagger}\left(s^{\prime}\right) \hat{a}_{n_{1}^{\prime}, k_{y 1}^{\prime}-q_{y 1}}^{\dagger}\left(s^{\prime}\right) \hat{a}_{n_{1}^{\prime}, k_{y_{1}}^{\prime}}\left(s^{\prime}\right)\right. \\
& \left.\times \hat{c}_{n_{1}, k_{y 1}}\left(s^{\prime}\right) \hat{c}_{n^{\prime}, k_{y}^{\prime}}^{\dagger}(\tau) \hat{c}_{n^{\prime}, k_{y}^{\prime}}(t) \hat{c}_{n_{2}, k_{y 2}+q_{y 2}}^{\dagger}\left(t^{\prime}\right) \hat{a}_{n_{2}^{\prime}, k_{y_{2}}^{\prime}-q_{y^{2}}}^{\dagger}\left(t^{\prime}\right) \hat{a}_{n_{2}^{\prime}, k_{y_{2}}^{\prime}}\left(t^{\prime}\right) \hat{c}_{n_{2}, k_{y 2}}\left(t^{\prime}\right) \hat{c}_{n, k_{y}}^{\dagger}(0)\right\rangle \\
= & \theta(s) \theta(t) \sum_{n_{i}, n_{i}^{\prime}, k_{y i}, k_{y i}^{\prime}, q_{i}} \int_{\tau-s}^{\tau} d s^{\prime} \int_{0}^{t} d t^{\prime} v_{q_{1}} v_{q_{2}} C_{n_{1} n_{1}^{\prime}}\left(q_{1}\right) C_{n_{2} n_{2}^{\prime}}\left(q_{2}\right) \\
& \times\left\langle\hat{a}_{n_{1}^{\prime}, k_{y 1}^{\prime}-q_{y 1}}^{\dagger}\left(s^{\prime}\right) \hat{a}_{n_{1}^{\prime}, k_{y_{1}}^{\prime}}\left(s^{\prime}\right) \hat{a}_{n_{2}^{\prime}, k_{y 2}^{\prime}-q_{y_{2}}}^{\dagger}\left(t^{\prime}\right) \hat{a}_{n_{2}^{\prime}, k_{y 2}^{\prime}}\left(t^{\prime}\right)\right\rangle \\
& \times\left\langle\hat{c}_{n, k_{y}}(\tau-s) \hat{c}_{n_{1}, k_{y 1}+q_{y 1}}^{\dagger}\left(s^{\prime}\right) \hat{c}_{n_{1}, k_{y 1}}\left(s^{\prime}\right) \hat{c}_{n^{\prime}, k_{y}^{\prime}}^{\dagger}(\tau) \hat{c}_{n^{\prime}, k_{y}^{\prime}}(t) \hat{c}_{n_{2}, k_{y 2}+q_{y 2}}^{\dagger}\left(t^{\prime}\right) \hat{c}_{n_{2}, k_{y 2}}\left(t^{\prime}\right) \hat{c}_{n, k_{y}}^{\dagger}(0)\right\rangle \\
= & \theta(s) \theta(t) \sum \sum_{n_{i}, n_{i}^{\prime}, k_{y 1}, k_{y 2}^{\prime}, q_{i}} \int_{\tau-s}^{\tau} d s^{\prime} \int_{0}^{t} d t^{\prime}\left\langle T \rho\left(q_{1}, s^{\prime}\right) \rho\left(q_{2}, t^{\prime}\right)\right\rangle v_{q_{1}} v_{q_{2}} \delta\left(\mathbf{q}_{1}+\mathbf{q}_{2}\right) F_{n_{1} n_{1}^{\prime}}\left(q_{1}\right) F_{n_{2} n_{2}^{\prime}}\left(q_{2}\right) \\
& \times\left\langle\hat{c}_{n, k_{y}}(\tau-s) \hat{c}_{n_{1}, k_{y 1}+q_{y 1}}^{\dagger}\left(s^{\prime}\right) \hat{c}_{n_{1}, k_{y 1}}\left(s^{\prime}\right) \hat{c}_{n^{\prime}, k_{y}^{\prime}}^{\dagger}(\tau) \hat{c}_{n^{\prime}, k_{y}^{\prime}}(t) \hat{c}_{n_{2}, k_{y 2}+q_{y 2}}^{\dagger}\left(t^{\prime}\right) \hat{c}_{n_{2}, k_{y 2}}\left(t^{\prime}\right) \hat{c}_{n, k_{y}}^{\dagger}(0)\right\rangle
\end{aligned}
$$

In the above equation, we have used the following definition for the density operator:

$$
\rho(q, \tau)=\sum_{n n^{\prime} k_{y}} F_{n n^{\prime}}(q) c_{n, k_{y}+q_{y}}^{\dagger}(\tau) c_{n^{\prime}, k_{y}}(\tau),
$$

where $F_{n n^{\prime}}(q)$ is related to $C_{n n^{\prime}}(q)$ via $C_{n n}(q)=\left|F_{n n^{\prime}}(q)\right|^{2}$. In the following, we shall assume that the emitter is also a pure two-dimensional system. In this case $v_{q}=\left(2 \pi e^{2} / q\right) e^{-q d}$, where $d$ is the separation between the emitter and the resonant well.

Now we note that $\left\langle\rho\left(q, s^{\prime}\right) \rho\left(-q, t^{\prime}\right)\right\rangle$ depends only on the difference between $s^{\prime}$ and $t^{\prime}$, that is $\left\langle\rho\left(q, s^{\prime}\right) \rho\left(-q, t^{\prime}\right)\right\rangle=$ $\left\langle\rho\left(q, s^{\prime}-t^{\prime}\right) \rho(-q, 0)\right\rangle$. The density-density correlation function can be related to the dielectric function of the electron gas via the fluctuation and dissipation theorem, ${ }^{15}$

$$
\left\langle T_{u} \rho(q, u) \rho(-q, 0)\right\rangle=\frac{-q}{2 \pi^{2} e^{2}} \int_{\infty}^{\infty} d \omega e^{-\imath \omega u} \frac{1}{1-e^{-\beta \omega}} \operatorname{Im}\left[\frac{1}{\epsilon(q, \omega)}\right] .
$$

We note that $1 /\left(1-e^{-\beta \omega}\right)=N(-\omega)=1+N(\omega)$, where $N(\omega)$ is the boson distribution function. The second order Green's function can now be written as

$$
\begin{aligned}
G_{\alpha, \alpha^{\prime}}^{(2)}(\tau, s, t)= & \frac{\theta(s) \theta(t)}{\pi} \sum_{\alpha_{1}, \alpha_{1}^{\prime} ; \alpha_{2}, \alpha_{2}^{\prime}} \frac{2 \pi e^{2}}{q} e^{-2 q d} F_{n_{1} n_{1}^{\prime}}\left(q_{1}\right) F_{n_{2} n_{2}^{\prime}}\left(q_{2}\right) \int_{-\infty}^{\infty} d \omega\left[1+N_{q}(\omega)\right] \operatorname{Im}\left[\frac{1}{\epsilon(q, \omega)}\right] \\
& \times \int_{\tau-s}^{\tau} d s^{\prime} \int_{0}^{t} d t^{\prime}\left\langle\hat{c}_{\alpha}(\tau-s) \hat{c}_{\alpha_{1}}^{\dagger}\left(s^{\prime}\right) \hat{c}_{\alpha_{1}^{\prime}}\left(s^{\prime}\right) \hat{c}_{\alpha^{\prime}}^{\dagger}(\tau) \hat{c}_{\alpha^{\prime}}(t) \hat{c}_{\alpha_{2}}^{\dagger}\left(t^{\prime}\right) \hat{c}_{\alpha_{2}^{\prime}}\left(t^{\prime}\right) \hat{c}_{\alpha}^{\dagger}(0)\right\rangle e^{-\imath \omega\left(s^{\prime}-t^{\prime}\right)},
\end{aligned}
$$


where $\alpha_{1}=\left(n_{1}, k_{y 1}\right), \alpha_{1}^{\prime}=\left(n_{1}, k_{y 1}+q_{y}\right), \alpha_{2}=\left(n_{2}, k_{y 2}\right), \alpha_{2}^{\prime}=\left(n_{2}, k_{y 2}-q_{y}\right)$. The last factor in Eq. (12) including the $s^{\prime}$ and the $t^{\prime}$ integration can be evaluated. The $\left\langle\hat{c}^{\prime} s\right\rangle$ term can be arranged into three combinations of four Green's functions [given by Figs. 2(b)-2(d)]. The integration is then completed after expanding these Green's functions in the wide band limit.

$$
\begin{aligned}
& G_{\alpha}^{1}(\tau, s, t)=\frac{G_{\alpha}^{0}(\tau, s, t)}{\left(\omega-\Delta_{n n^{\prime}}\right)^{2}} e^{\imath \Delta_{n n^{\prime}}(t-s)} e^{-\imath \omega_{n n^{\prime}}(\tau-s)}\left(e^{-\imath\left(\omega-\Delta_{n n^{\prime}}\right) s}-1\right)\left(e^{\imath\left(\omega-\Delta_{n n^{\prime}}\right) t}-1\right) \\
& G_{\alpha}^{2}(\tau, s, t)=G_{\alpha}^{0}(\tau, s, t)\left\{\frac{\imath t}{\omega-\Delta_{n n^{\prime}}}+\frac{e^{-\imath\left(\omega+\Delta_{n n^{\prime}}\right) t}-1}{\left(\omega+\Delta_{n n^{\prime}}\right)^{2}}\right\} \\
& G_{\alpha}^{3}(\tau, s, t)=G_{\alpha}^{0}(\tau, s, t)\left\{\frac{-\imath s}{\omega-\Delta_{n n^{\prime}}}+\frac{e^{\imath\left(\omega+\Delta_{n n^{\prime}}\right) s}-1}{\left(\omega+\Delta_{n n^{\prime}}\right)^{2}}\right\}
\end{aligned}
$$

where $\Delta_{n n^{\prime}}=\left(n-n^{\prime}\right) \hbar \omega_{c}$. Now we can apply exponential resummation $G_{\alpha}(\tau, s, t)=G_{\alpha}^{0}(\tau, s, t) \exp \left[\left(G^{1}+G^{2}+G^{3}\right) / G^{0}\right]$ to obtain

$$
\begin{aligned}
T_{\text {tot }}\left(\varepsilon_{n}\right)= & \frac{\Gamma_{L} \Gamma_{R}}{\Gamma} \int_{-\infty}^{\infty} d \sigma \exp \left[\frac{-\Gamma|\sigma|}{2}+\imath\left(\varepsilon_{k_{z}}-\epsilon_{c}\right) \sigma+\sum_{q, n^{\prime}} \frac{2 e^{2}}{q} e^{-2 q d} C_{n n^{\prime}}(q)\right. \\
& \left.\times \int_{-\infty}^{\infty} d \omega[1+N(\omega)] \operatorname{Im}\left[\frac{1}{\epsilon(q, \omega)}\right]\left(\frac{\imath \sigma}{\left(\omega-\Delta_{n n^{\prime}}\right)}+\frac{e^{-\imath\left(\omega-\Delta_{n n^{\prime}}\right) \sigma}-1}{\left(\omega-\Delta_{n n^{\prime}}\right)^{2}}\right)\right] .
\end{aligned}
$$

If the dynamics of the electron-electron interaction is ignored, $\operatorname{Im}(1 / \epsilon(q, 0)) \equiv 0$, we immediately recover the well known resonant tunneling result,

$$
T_{\mathrm{tot}}=\frac{\Gamma_{L} \Gamma_{R}}{\left(\epsilon_{z}-\epsilon_{c}\right)^{2}+\Gamma^{2} / 4}
$$

The term proportional to $e^{2} \operatorname{Im}(1 / \epsilon(q, \omega))$ contains all contributions to the total tunneling rate, due to electronic excitations of the system. In the plasmon pole approximation, $\operatorname{Im}(1 / \epsilon(q, \omega)) \propto \delta\left(\omega \pm \omega_{p}\right)$, where $\omega_{p}$ is the plasma frequency, we recover our previous result of plasmon assisted tunneling. ${ }^{8}$ The effect of plasmon excitation on resonant tunneling was treated in Ref. 8 by introducing an additional set of boson coordinates. The derivation presented here shows that such electronic excitation assisted tunneling can be studied within the framework of an elementary electron-electron interaction. The boson coordinates describing the collective excitation are completely redundant.

The dielectric function appearing in the above equation depends on the magnetic field. For an infinitely sharp LL, $\operatorname{Im}[1 / \epsilon]$ only contains contributions from magnetoplasmons, i.e.,

$$
\operatorname{Im} \frac{1}{\epsilon(q, \omega)}=\pi \delta(\operatorname{Re} \epsilon(q, \omega))=\pi \delta\left(1-\sum_{m} \frac{b_{m} \omega_{c}^{2}}{\omega^{2}-m^{2} \omega_{c}^{2}}\right)
$$

and

$$
b_{m}=4 m r_{s} \frac{k_{F}}{q} X^{m} e^{-X} \sum_{m^{\prime}} \frac{m^{\prime} !}{\left(m+m^{\prime}\right) !}\left[L_{m^{\prime}}^{m}(X)\right]^{2}\left[f_{m^{\prime}}-f_{m+m^{\prime}}\right]
$$

where $X=(q \hbar)^{2} /(2 e B)$, and $r_{s}=m e^{2} / k_{F}$ is the plasma parameter and $f_{m}$ is the Fermi distribution function. In general, there are countably infinite collective modes. However, the coupling to the higher mode decreases as $1 / m$ and we shall only include the first few modes in our model calculation below. Furthermore, we shall assume that the mixing of different modes is negligible, i.e., the $m$ th mode is given as

$$
\omega_{p}^{(m \pm)}= \pm \omega_{c} \sqrt{b_{m}+m^{2}} .
$$

Therefore, the total tunneling rate can be written as

$$
\begin{aligned}
T_{\mathrm{tot}}\left(\varepsilon_{n}\right)= & \frac{\Gamma_{L} \Gamma_{R}}{\Gamma} \int_{-\infty}^{\infty} d \sigma \exp \left[\frac{-\Gamma|\sigma|}{2}+\imath\left(\varepsilon_{k_{\perp}}-\epsilon_{c}\right) \sigma+\sum_{q, n^{\prime}, m} \frac{2 \pi e^{2}}{q} C_{n n^{\prime}}(q) \frac{b_{m} \omega_{c}^{2}}{\omega_{p}^{(m+)}} e^{-2 q d}\right. \\
& \left.\times\left\{\left[1+N\left(\omega_{p}^{(m+)}\right)\right] F\left(q, \omega_{p}^{(m+)}\right)+N\left(\omega_{p}^{(m+)}\right) F\left(q, \omega_{p}^{(m-)}\right)\right\}\right]
\end{aligned}
$$



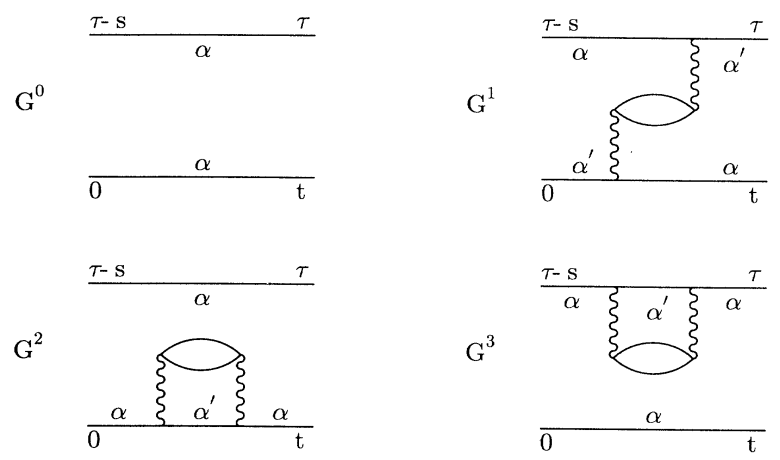

FIG. 2. A class of diagrams, which contribute to the second order two-body Green's functions. Here, a straight line with index $\alpha$ represents the single particle Green's function $G_{\alpha}$ and a wavy line represents the electron-electron interaction $V_{\alpha \alpha^{\prime}}=v_{q} C_{n n^{\prime}}(q)$, a bubble represents the densitydensity response.

where

$F\left(q, \omega_{p}^{(m+)}\right)=\frac{\imath \sigma}{\left(\omega_{p}^{(m+)}-\Delta_{n n^{\prime}}\right)}+\frac{e^{-\imath\left(\omega_{p}^{(m+)}-\Delta_{n n^{\prime}}\right) \sigma}-1}{\left(\omega_{p}^{(m+)}-\Delta_{n n^{\prime}}\right)^{2}}$.

In Eq. (16), two terms inside the curly bracket have direct physical meaning. The first term, which is proportional to $[1+N(\omega)]$, represents the tunneling process assisted by emission of magnetoplasmons, while the second term, which is proportional to $N(\omega)$, represents the tunneling process assisted by magnetoplasmon absorption. At low temperature, $N \ll 1$ and the absorption process can be neglected.

The current is then given as

$$
I=e \sum_{n} T_{\text {tot }}\left(\epsilon_{n}\right) g_{n}\left[f_{e}\left(\epsilon_{\mathbf{p}}\right)-f_{c}\left(\epsilon_{\mathbf{p}}+e V\right)\right],
$$

where $g_{n}$ is the level degeneracy and $f_{e}\left(f_{c}\right)$ is the Fermi distribution function of the emitter (collector).

\section{RESULTS AND DISCUSSIONS}

We have calculated the tunneling current, with the use of Eqs. (16) and (18). The parameters used in our calculation are $E_{F}=5 \mathrm{meV}, d=100 \AA, \Gamma=1 \mathrm{meV}$, and $\epsilon_{z}-\left.\epsilon_{c}\right|_{\text {bias }=0}=15 \mathrm{meV}$. The result is plotted in Fig. 3. Only the first two magnetoplasmon modes are included. The resonant tunneling with plasmon emission can be clearly seen. For $B=1.2 \mathrm{~T}$, the first satellite at 16.7 $\mathrm{meV}$ corresponds to the emission of a $\omega^{(1)}$ plasmon, the next satellite at $19.2 \mathrm{meV}$ is due to emission of a $\omega^{(2)}$ plasmon. The emission of two $\omega^{(1)}$ plasmons can also be seen at around $22.1 \mathrm{meV}$. All higher order processes are too weak to see. As magnetic field increases, the magnetoplasmon satellites move towards the higher energy.

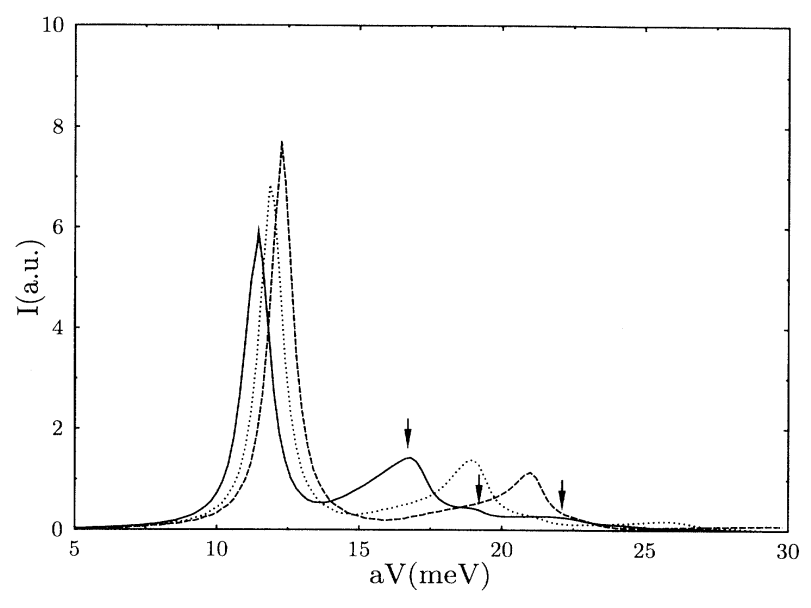

FIG. 3. Plot of tunneling current as a function of bias $V$ for different magnetic fields, $B=1.2 \mathrm{~T}$ (solid line, arrows indicate those plasmon processes described in the text); $B=$ $1.75 \mathrm{~T}$ (dotted line) and $B=2.3 \mathrm{~T}$ (broken line). Here, $d=100 \AA, \Gamma=1 \mathrm{meV}, a V$ is the portion of bias across the emitting barrier, and $a \approx 0.5$ for a symmetric DBRTS.

In the meantime, the main resonances become stronger and the satellites become weaker as the field strength is increased. Therefore, applying a parallel magnetic field will have two competing effects. On the one hand, the plasmon frequency is enhanced and the satellites become better resolved; on other hand, the real strength of the satellites is reduced. The spacing between the main resonance and the subsequent satellites is not proportional to $B$, due to the inclusion of coherence breaking processes, and more importantly, due to the $q$ dependence of $\omega_{p}$. If one neglects these coherence breaking processes $\left(n \neq n^{\prime}\right)$, the spacing will be close to linear in $B$, especially at large $B$. For a system with large emitter-well separation or large electron concentration in the emitter, the main contribution to the $q$-dependent part of $\omega_{p}$ is from the small $q$ regime. In this case, the spacing will also show close to linear behavior. Figure 4 shows the same calculation, but with a thicker emitter barrier, $d=200 \AA$ and $\Gamma=0.2 \mathrm{meV}$. As $d$ is increased, the electron-plasmon coupling becomes weaker. The magnetoplasmon assisted tunneling, which gives rise to additional satellites in Fig. 3, now only shows some weak shoulder structure in this case. The ratio of the main resonance to the first plasmon satellite in Fig. 3 is about 4 and in Fig. 4 is about 15. This rapid increase is mainly because the main resonance becomes much sharper as $\Gamma$ decreases. The electron-electron interaction is also reduced. However, this reduction is less important, because the dominant contribution comes from the small- $q$ regime and the main effect of $e^{-q d}$ is to cut off the contribution from the large- $q$ regime.

Now we compare briefly the magnetoplasmon emission mechanism with the elastic scattering mechanism. From this work, it is evident that due to the electron-electron 


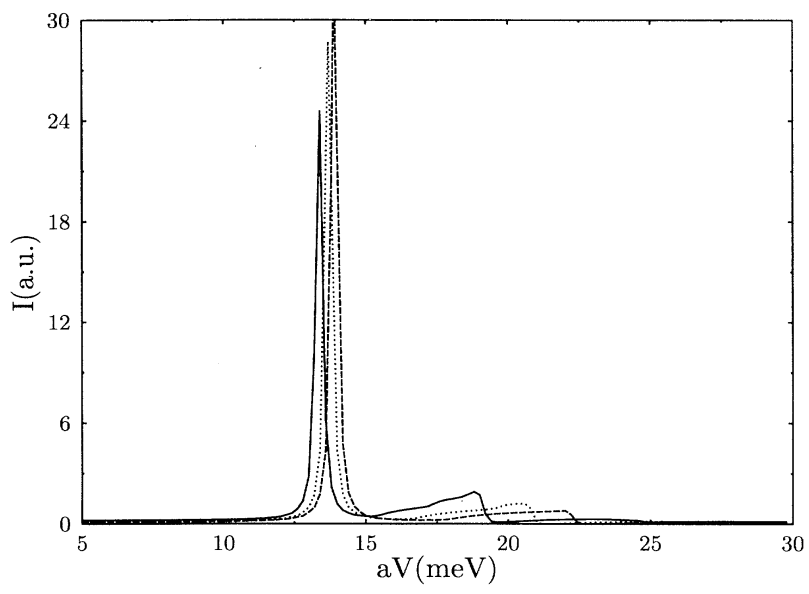

FIG. 4. Same as Fig. 3, but with $d=200 \AA$ and $\Gamma=0.2$ $\mathrm{meV}$.

interaction, magnetoplasmon excitation can open an additional channel for electron resonant tunneling through a DBRTS. However, unlike the case of inter-Landau-level elastic scattering, where a series of almost equal strength satellites were expected and observed,${ }^{9}$ here only the single plasmon emission gives rise to a distinct satellite resonance (if the electron-plasmon coupling is strong enough as in the case of Fig. 3) and all higher order plasmon ex- citations only give rise to weak shoulders to the tunneling current. This is mainly due to the fact that the magnetoplasmon energy is strongly dependent on the wave vector $q$ and magnetoplasmon assisted tunneling contains all contributions of different $q$ processes. Therefore, for samples with realistic disorder concentration, such as those studied in Ref. 9, the elastic scattering should still be the plausible mechanism for understanding the magnetoresonant tunneling (one does need to bear in mind that no low bias satellite having been observed experimentally requires further explanation). The magnetoplasmon assisted tunneling may become a plausible mechanism in a system where scatterers are negligible. We, therefore, propose that magnetotunneling measurements be performed in high mobility and low scattering samples.

In conclusion, we have studied a resonant tunneling phenomenon in a parallel magnetic field. It is found that due to electron magnetoplasmon coupling, side resonances or shoulders structures can appear on the high bias side of the main resonance at low temperature.

\section{ACKNOWLEDGMENTS}

We would like to thank P. E. Simmonds, A. D. Martin, M. L. F. Lerch, and L. Eaves for stimulating discussions. This work is supported in part by the Australian Research Council and University of Wollongong Postgraduate Scholarship Awards.
${ }^{1}$ R. Tsu and L. Esaki, Appl. Phys. Lett. 22, 562 (1973).

${ }^{2}$ T. C. L. G. Sollner et al., Appl. Phys. Lett. 43, 588 (1983).

${ }^{3}$ A. Zaslavski et al., Appl. Phys. Lett. 53, 1408 (1988); E. S. Alves et al., Electron. Lett. 24, 1190 (1988).

${ }^{4}$ T. C. L. G. Sollner, Phys. Rev. Lett. 59, 1622 (1987);

V. J. Goldman, ibid. 59, 1623; T. J. Foster et al., Phys. Rev. B 39, 6205 (1989)

${ }^{5}$ M. L. Leadbeater et al., J. Phys. Condens. Matter 1, 10605 (1989).

${ }^{6}$ A. D. Martin, M. L. F. Lerch, P. E. Simmonds, and L. Eaves, Appl. Phys. Lett. 64, 1248 (1994).

${ }^{7}$ M. L. F. Lerch, A. D. Martin, P. E. Simmonds, L. Eaves, and M. L. Leadbeater, Solid-State Electron. 37, 961 (1994).

${ }^{8}$ C. Zhang, M. L. F. Lerch, A. D. Martin, P. E. Simmonds, and L. Eaves, Phys. Rev. Lett. 72, 3397 (1994).
${ }^{9}$ Y. G. Gobato, F. Chevior, J. M. Berroir, P. Bois, Y. Guldner, J. Nagle, J. P. Vieren, and B. Vinter, Phys. Rev. B 43, 4843 (1991), and references therein.

${ }^{10}$ D. D. Coon, K. Bandara, and H. Zhao, Appl. Phys. Lett. 54, 2115 (1989).

${ }^{11}$ N. S. Wingreen, K. W. Jacobson, and J. Wilkins, Phys. Rev. Lett. 61, 1396 (1988)

${ }^{12}$ N. Zou and K. A. Chao, Phys. Rev. Lett. 69, 3224 (1992).

${ }^{13}$ G. D. Mahan, Many-Particle Physics (Plenum, New York, 1981).

${ }^{14}$ G. D. Mahan, Phys. Rev. 153, 882 (1967); D. L. Langreth, Phys. Rev. B 1, 471 (1970).

${ }^{15}$ P. M. Platzman and P. A. Wolff, Waves and Interactions in Solid State Plasmas (Academic, New York, 1973). 